\title{
Fully Coupled Poroelastic Peridynamic Formulation for Fluid-Filled Fractures
}

\author{
Selda Oterkus ${ }^{\mathrm{a} *}$, Erdogan Madenci ${ }^{\mathrm{b}}$ and Erkan Oterkus ${ }^{\mathrm{a}}$ \\ ${ }^{a}$ Department of Naval Architecture, Ocean and Marine Engineering, University of \\ Strathclyde, Glasgow, G4 OLZ, United Kingdom \\ ${ }^{\mathrm{b}}$ Department of Aerospace and Mechanical Engineering, The University of Arizona, \\ Tucson, AZ 85721, United States
}

\begin{abstract}
A new fully coupled poroelastic peridynamic formulation is presented and its application to fluid-filled fractures is demonstrated. This approach is capable of predicting porous flow and deformation fields and their effects on each other. Moreover, it captures the fracture initiation and propagation in a natural way without resorting to an external failure criterion. The peridynamic predictions are verified by considering two benchmark problems including one- and two-dimensional consolidation problems. Moreover, the growth of a pre-existing hydraulically pressurized crack case is presented. Based on these results, it is concluded that the current peridynamic formulation has a potential to be used for the analysis of more sophisticated poroelastic problems including fluid-filled rock fractures as in hydraulic fracturing.
\end{abstract}

Keywords: peridynamics; poroelasticity; hydraulic; fracture

\section{Introduction}

The coupling between changes in stress and changes in fluid pressure forms the foundation of the subject of poroelasticity (Wang, 2000). The theory of poroelasticity has important applications in geomechanics including oil exploration, gas-hydrate detection, seismic monitoring of $\mathrm{CO}_{2}$ storage, hydrogeology, etc. (Carcione et al., 2010). Poroelasticity has been studied for the last one hundred fifty years. The increase of exploitation of groundwater and hydrocarbon resources during early $20^{\text {th }}$ century gave

*Corresponding author. Tel.: +44 (0)141548 4979.

E-mail addresses: selda.oterkus@strath.ac.uk (S. Oterkus), madenci@email.arizona.edu (E. Madenci), erkan.oterkus@strath.ac.uk (E. Oterkus) 
impetus to poroelastic research (Wang, 2000). Original formulation of poroelasticity was developed by Terzaghi (1925) based on his one-dimensional laboratory experiments and is known as Terzaghi's consolidation theory. This formulation was extended to a general three-dimensional formulation by Biot (1941). Numerical approaches have been widely used in the solution of poroelastic equations. Amongst these numerical techniques, finitedifference, psuedospectral, low-order finite element and spectral-finite element methods can be cited.

A recent popular application of poroelasticity is the analysis of hydraulic fracturing. The hydraulic fracturing process creates and propagates cracks in a porous medium by injecting fluid pressure. It has gained significant attention as a result of its use in oil and gas extraction from unconventional shale resources. In this particular application, a mixture of hydraulic fluid, sand, and chemicals is pumped into a well to create cracks in low-permeable shale, and keep them open after the removal of the fluid. Once the process is complete, the permeability of the shale increases significantly; thus, oil and/or gas starts to flow through the well. Another application of hydraulic fracturing concerns heat extraction from geothermal resources. Similar to the technique used in oil and gas extraction, the permeability of hot rocks is enhanced by pumping cold water into the rock. Cold water can be pumped from one well (injection well), and hot water can be extracted from the other well (production well). This technique, known as Hot Dry Rock (HDR), is used in the production of electricity.

It is difficult to model the hydraulic fracturing process due to the presence of multiple physical mechanisms; it involves fluid flow in a porous medium, mechanical deformation as a result of fluid pressure, and crack initiation and propagation. Various numerical models exist for the simulation of hydraulic fracturing. They are based on the Finite Element Method (FEM), Cohesive Zone Elements (CZE), eXtended FEM (XFEM), and, most recently, peridynamics. Hunsweck et al. (2013) developed a FEA-based algorithm, and captured the nonlinear coupling between the fluid pressure and crack opening. They included the fluid lag (the gap between the fluid front and the crack tip), which can be important for small toughness, and used the Griffith criterion for crack propagation. In another study, Ouyang et al. (1997) performed FEA for coupled hydraulic fluid transport 
and fracture analysis. They used an automatic and domain-adaptive remeshing technique for crack propagation.

Remeshing can be numerically difficult for certain problems; thus, CZE can be used to model hydraulic fracturing. Chen (2012) used a CZE to simulate the propagation of a viscosity-dominated hydraulic fracture in an infinite and impermeable elastic medium. Two different meshing schemes were utilized. The first scheme employed much finer cohesive elements than those of the neighboring elements. The second scheme employed the cohesive elements comparable in size with those of neighboring regular elements. The top and bottom faces of the cohesive elements were tied to the surrounding regular elements by imposing a surface-based tie constraint. As expected, the first scheme was computationally more expensive than the second scheme. It was reported that the accuracy did not depend on the size of the cohesive element.

This situation may cause a numerical problem in modeling the pressure distribution near the injection point and the crack tip for a viscosity-dominated hydraulic fracture because the fluid pressure changes rapidly at these locations. Although cohesive zone elements are easy to use for crack propagation, the method leads to a mesh dependency problem since the crack must follow the regular element boundaries. In order to overcome this problem, an extended finite element method (XFEM) was introduced and also used for hydraulic fracturing simulations. Lecampion (2009) utilized XFEM by introducing special tip functions to capture the crack tip asymptotic accurately. Both toughness-dominated and viscosity-dominated conditions were investigated; a difficulty was encountered in simulating large toughness and small viscosity conditions when the fracture was driven by a very small pressure gradient. In addition to FEM based approaches, meshless techniques are also developed for fluid-driven fracture such as immersed particle method (Rabczuk et al., 2010a). In this technique, structure and fluid particles are coupled by using a master-slave scheme and fluid is allowed to flow through openings between crack surfaces.

As opposed to the aforementioned studies that are mainly based on classical continuum mechanics, the hydraulic fracturing phenomenon can also be analyzed by using peridynamics (Silling, 2000), which is a non-local continuum mechanics approach. Peridynamics removes the difficulties that were observed in earlier studies, such as mesh 
dependency, unphysical singularities, and selection of a suitable failure criterion. Moreover, the inherent characteristic of peridynamics permits crack propagation in a natural way. Hence, Turner (2013) extended the original form of peridynamics by including the fluid pore pressure. However, the pore pressure was calculated by using a local approach a priori. On the other hand, this study presents a fully coupled bond based peridynamics approach to simultaneously simulate both deformation and porous flow fields. The current peridynamic formulation has a potential to be used for the analysis of more sophisticated poroelastic problems including fluid-filled rock fractures as in hydraulic fracturing and presented in Zhou and Burbey (2014), Li. et. al. (2014) and Helmons et al. (2016). Moreover, the formulation can be extended to be applicable for more complex material behavior by following a similar procedure explained in Oterkus and Madenci (2014).

\section{Peridynamics}

A non-local continuum theory, Peridynamics, introduced by Silling (2000) overcomes the aforementioned difficulties arising due to the existence of discontinuities in the structure. As opposed to classical continuum mechanics, a material point inside the body can interact with other material points within its domain of influence called the "horizon," $H$ with radius $\delta$ as shown in Fig. 1 The interaction (bond) between two material points $\mathbf{x}$ and $\mathbf{x}^{\prime}$ is expressed by using a response function, $\mathbf{f}$. The response function contains all the constitutive information related to the material point. It is assumed that the interactions beyond the horizon vanish. Although the original application of peridynamics concerns the equation of motion and cracking in an elastic medium, it is also applicable to other field equations (Gerstle et al., 2008; Oterkus and Madenci, 2011, 2013, 2014; Oterkus, et al., 2013, 2014a, 2014b, 2014c; Oterkus, 2015;

Han et al., 2015, 2016; Kilic and Madenci, 2010; De Meo et al., 2016; Bobaru and Duangpanya, 2010, 2012; Chen and Bobaru, 2015). The detailed derivation of peridynamics and its applications are given in a book by Madenci and Oterkus (2014). 


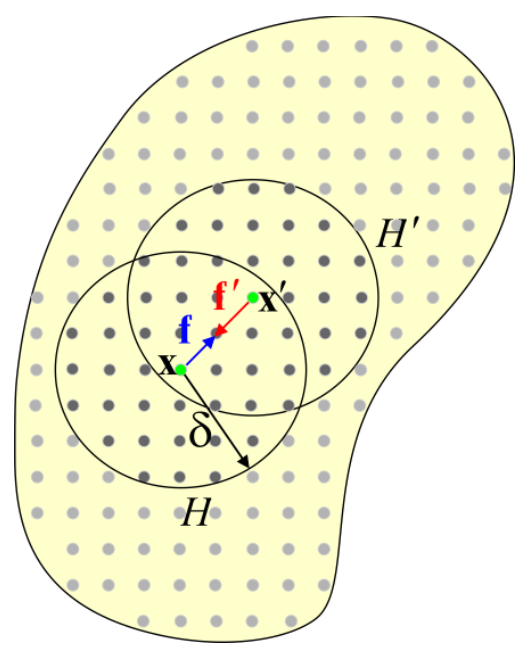

Fig. 1. Interaction of a material point with its neighboring points.

\subsection{Peridynamic equation of motion}

As opposed to the classical continuum mechanics, displacement derivatives do not appear in peridynamic (PD) equations, allowing the peridynamic formulation to hold everywhere whether or not displacement discontinuities are present. As derived by Silling (2000), the peridynamic equation of motion at a reference position of $\mathbf{x}$ and time $t$ is given as

$$
\rho(\mathbf{x}) \mathbf{u}(\mathbf{x}, t)=\int_{H} \mathbf{f}\left(\mathbf{x}^{\prime}-\mathbf{x}, \mathbf{u}^{\prime}-\mathbf{u}\right) d V+\mathbf{b}(\mathbf{x}, t)
$$

in which $\mathbf{u}$ is the displacement vector field, $\mathbf{b}$ is a prescribed body-force density field, and $\rho$ is mass density in the reference configuration. The response function, $\mathbf{f}$, that defines the force between two material points is written as

$$
\mathbf{f}=c s \frac{\mathbf{y}^{\prime}-\mathbf{y}}{\left|\mathbf{y}^{\prime}-\mathbf{y}\right|}
$$

in which $\mathbf{y}$ represents the position of the material point, $\mathbf{x}$ in the deformed configuration. Also, the stretch, $s$, between the material points is defined as 
$s=\frac{\left|\mathbf{y}^{\prime}-\mathbf{y}\right|-\left|\mathbf{x}^{\prime}-\mathbf{x}\right|}{\left|\mathbf{x}^{\prime}-\mathbf{x}\right|}$

As derived by Silling and Askari (2005), the material parameter (bond constant), $c$, for an isotropic material can be expressed as

$$
\begin{aligned}
& c=\frac{2 E}{A \delta^{2}}(1-\mathrm{D}) \\
& c=\frac{9 E}{\pi h \delta^{3}}(2-\mathrm{D}) \\
& c=\frac{12 E}{\pi \delta^{4}}(3-\mathrm{D})
\end{aligned}
$$

in which $E$ represents the Young's modulus of the solid skeleton, and $A$ and $h$ are the cross-sectional area and thickness, respectively.

\subsubsection{Mechanical deformation due to fluid flow}

There exists a complete analogy between poroelasticity and thermoelasticity (Wang, 2000). Therefore, in the presence of a fluid presure, the peridynamic equation of motion of a material point at $\mathbf{x}$ can be modified as

$$
\rho \mathbf{u}(\mathbf{x}, t)=\int_{H} c\left(s-\alpha_{B} \gamma \bar{P}\right) \frac{\mathbf{y}^{\prime}-\mathbf{y}}{\left|\mathbf{y}^{\prime}-\mathbf{y}\right|} d V+\mathbf{b}(\mathbf{x}, t)
$$

in which $\alpha_{B}$ is the Biot parameter, and $\gamma$ is the coefficient of fluid pore pressure defined as

$$
\begin{aligned}
& \gamma=\frac{1}{E}(1-\mathrm{D}) \\
& \gamma=\frac{(1-v)}{E}(2-\mathrm{D})
\end{aligned}
$$




$$
\gamma=\frac{(1-2 v)}{E}(3-\mathrm{D})
$$

with $v$ representing the Poisson's ratio. The average fluid pore pressure, $\bar{P}$, of material points $\mathbf{x}$ and $\mathbf{x}^{\prime}$ is defined as

$$
\bar{P}=\frac{P\left(\mathbf{x}^{\prime}, t\right)+P(\mathbf{x}, t)}{2}
$$

\subsubsection{Fluid flow in porous media}

The governing equations for a single-phase fluid flow in a porous medium can be described by Darcy's Law as (Biot, 1941)

$$
\frac{\partial\left(\rho_{f} \phi\right)}{\partial t}=-\nabla \cdot\left(\rho_{f} \mathbf{v}\right)+q_{f}
$$

where $\phi$ is the variation in the water content, $\rho_{f}$ is the density of the fluid, and $q_{f}$ is the mass of fluid produced per unit volume per unit time. The fluid velocity, $\mathbf{v}$, can be expressed as

$$
\mathbf{v}=-\frac{k_{P}}{\mu_{v}} \nabla P
$$

with

$$
P=p_{f}-\rho_{f} g z
$$

and $p_{f}$ is the fluid pressure, $k_{P}$ is the permeability of the porous medium, $\mu_{v}$ is the fluid viscosity, $g$ is the magnitude of the gravitational acceleration, and $z$ is the depth. The variation in the water content can be defined as 


$$
\phi=\alpha_{B} \theta+\frac{P}{Q_{B}}
$$

where $Q_{B}, \alpha_{B}$, and $\theta$ represent the Biot modulus, Biot parameter, and the change in volume (dilatation), respectively.

As derived by Biot (1941), Eq. (8) can be rewritten for constant material properties as

$$
\frac{1}{Q_{B}} \frac{\partial P}{\partial t}=\frac{k_{P}}{\mu} \nabla^{2} P-\alpha_{B} \frac{\partial \theta}{\partial t}+\frac{q_{f}}{\rho_{f}}
$$

By using the analogy between poroelasticity and thermoelasticity (Oterkus et al., 2016a), the peridynamic form of Eq. (11) can be written as

$$
\frac{1}{Q_{B}} P(\mathbf{x}, t)=\int_{H}\left(\kappa_{P} \frac{P\left(\mathbf{x}^{\prime}, t\right)-P(\mathbf{x}, t)}{\left|\mathbf{x}^{\prime}-\mathbf{x}\right|}-\frac{c}{2} \alpha_{B} \gamma e\right) d V+\frac{q_{f}}{\rho_{f}}
$$

in which the peridynamic parameter, $\kappa_{P}$, can be expressed in terms of the permeability of the bulk material, $k_{P}$, and the fluid viscosity, $\mu_{v}$, as

$$
\kappa_{P}=\frac{2 k_{P}}{\mu_{v} A \delta^{2}}(1-\mathrm{D}), \kappa_{P}=\frac{6 k_{P}}{\pi \mu_{v} h \delta^{3}}(2-\mathrm{D}), \kappa_{P}=\frac{6 k_{P}}{\pi \mu_{v} \delta^{4}}(3-\mathrm{D})
$$

Based on the definition of extension between the material points, i.e.

$$
e=\left|\mathbf{y}^{\prime}-\mathbf{y}\right|-\left|\mathbf{x}^{\prime}-\mathbf{x}\right|
$$

the time rate of change of extension can be expressed as

$$
e=\frac{\mathbf{y}^{\prime}-\mathbf{y}}{\left|\mathbf{y}^{\prime}-\mathbf{y}\right|} \cdot\left(\mathbf{u}^{\prime}-\mathbf{u}\right)
$$


Moreover, the peridynamic form of the fluid velocity can be expressed as

$$
\mathbf{v}=-\frac{1}{2} \int_{H} \kappa_{P}\left[P\left(\mathbf{x}^{\prime}, t\right)-P(\mathbf{x}, t)\right] \frac{\mathbf{x}^{\prime}-\mathbf{x}}{\left|\mathbf{x}^{\prime}-\mathbf{x}\right|} d V
$$

\subsection{Boundary conditions}

Unlike the local theory, the boundary conditions in peridynamics can be defined through a nonzero volume of fictitious boundary layers. Based on the study by Macek and Silling (2007), the size of the fictitious layer can be chosen as the horizon size, $\delta$. The flow boundary conditions can be imposed by assigning constraint conditions to the material points in the fictitious layer. No flux boundary condition can be imposed by using mirror images of the pressure values of material points adjacent to the boundary in the actual domain for the material points in the fictitious region (Oterkus and Madenci, 2015). Displacement boundary conditions can also be imposed similarly (Madenci and Oterkus, 2016). The traction boundary conditions can be imposed as body force in the fictitious region (Madenci and Oterkus, 2014). On the other hand, the pressure boundary conditions on the crack surfaces can be imposed by simply assigning their values to the material points nearest to the crack surface.

\section{Numerical procedure}

Fully coupled poroelastic peridynamic system of equations can be solved numerically by using staggered strategy (Armero and Simo, 1992; Farhat et al., 1991; Liu and Chang, 1985). Therefore, the equation of motion is solved for the displacement field and the porous flow equation is solved for the fluid pore pressure field.

Eq. (5) and (12) can be discretized by using meshless scheme as

$$
\rho \mathbf{u}_{(i)}^{n}=\sum_{j=1}^{N} c\left(s_{(i)(j)}^{n}-\alpha_{B} \gamma \bar{P}_{(i)(j)}^{n}\right) \frac{\mathbf{y}_{(j)}^{n}-\mathbf{y}_{(i)}^{n}}{\left|\mathbf{y}_{(j)}^{n}-\mathbf{y}_{(i)}^{n}\right|} V_{(j)}+\mathbf{b}_{(i)}
$$

and 


$$
\frac{1}{Q_{B}} P_{(i)}^{n}=\sum_{j=1}^{N}\left(\kappa_{P} \frac{P_{(j)}^{n}-P_{(i)}^{n}}{\left|\mathbf{x}_{(j)}-\mathbf{x}_{(i)}\right|}-\frac{c}{2} \alpha_{B} \gamma e_{(i)(j)}^{n}\right) V_{(j)}+\frac{q_{f(i)}}{\rho_{f(i)}}
$$

Here $n$ represents the time step number, $j$ represents the family members of material point $i$, and $V_{(j)}$ represents the volume of the material point. The average fluid pore pressure, time rate of exchange of extension and stretch can be written by using Eqs. (7), (15) and (3) as

$$
\begin{aligned}
& \bar{P}_{(i)(j)}^{n}=\frac{P_{(j)}^{n}+P_{(i)}^{n}}{2} \\
& e_{(i)(j)}^{n}=\frac{\mathbf{y}_{(j)}^{n}-\mathbf{y}_{(i)}^{n}}{\left|\mathbf{y}_{(j)}^{n}-\mathbf{y}_{(i)}^{n}\right|} \cdot\left(\mathbf{u}_{(j)}^{n}-\mathbf{u}_{(i)}^{n}\right) \\
& s_{(i)(j)}^{n}=\frac{\left|\mathbf{y}_{(j)}^{n}-\mathbf{y}_{(i)}^{n}\right|-\left|\mathbf{x}_{(j)}^{n}-\mathbf{x}_{(i)}^{n}\right|}{\left|\mathbf{x}_{(j)}^{n}-\mathbf{x}_{(i)}^{n}\right|}
\end{aligned}
$$

If the stretch value given in Eq. (19c) exceeds a critical stretch value, then the interaction between material points $i$ and $j$ is broken. Note that this type of definition of failure is different than other meshless techniques including cracking-particle method where the crack is modelled by using a discontinuous enrichment function (Rabczuk and Belytschko, 2004, 2007) or the particles are split into two particles lying on opposite sides of the crack (Rabczuk et al., 2010b). Moreover, a uniform discretization is used throughout this study. An adaptive refinement scheme can be used by using DualHorizon Peridynamics (Ren et al., 2016) which has an advantage of minimal artificial wave reflection.

Time integration can be achieved by using explicit time integration scheme for the solution of coupled equations. The explicit time integration schemes explained in Silling and Askari (2005) and Oterkus et al. (2014b) can be utilized for the solution of the 
displacement field and the porous flow equation, respectively. Since explicit time integration scheme is a conditional technique, the critical time step size can be chosen as the smaller critical time step size of the displacement field and porous flow equation.

\section{Numerical results}

In order to demonstrate the capability of the current formulation, first, a onedimensional fully coupled consolidation problem, whose analytical solution (Wang, 2000; Biot, 1941) exists, is considered. Then, this problem is extended to include the second dimension, and PD predictions are compared against finite element analysis results. After establishing the validity of the PD coupled field equations, growth of a hydraulically pressurized crack in a two-dimensional region is demonstrated.

\subsection{One-dimensional consolidation}

This problem, considered previously by Biot (1941) and Wang (2000), concerns the determination of the pore pressure, $P(z, t)$, and displacement, $u_{z}(z, t)$, of a fluid-saturated region. These two fields are fully coupled. The one-dimensional region with length $L=15 \mathrm{~m}$ (Fig. 2) is subjected to a constant pressure of $P_{o}=1 \times 10^{4} \mathrm{~N} / \mathrm{m}^{2}$ at one end $(z=0)$, and fixed at the other end $(z=L)$.

The elastic modulus and Poisson's ratio of the porous material are specified as $E=1.0 \times 10^{8} \mathrm{~N} / \mathrm{m}^{2}$ and $v=1 / 3$, respectively. The Biot modulus and Darcy conductivity of the material are specified as $Q=1 / 1.65 \times 10^{-10} \mathrm{~N} / \mathrm{m}^{2}$ and $\kappa=k_{p} / \mu=1.02 \times 10^{-9} \mathrm{~m}^{4} / \mathrm{Ns}$, respectively. The density of the material is specified as $\rho=1900 \mathrm{~kg} / \mathrm{m}^{3}$. 


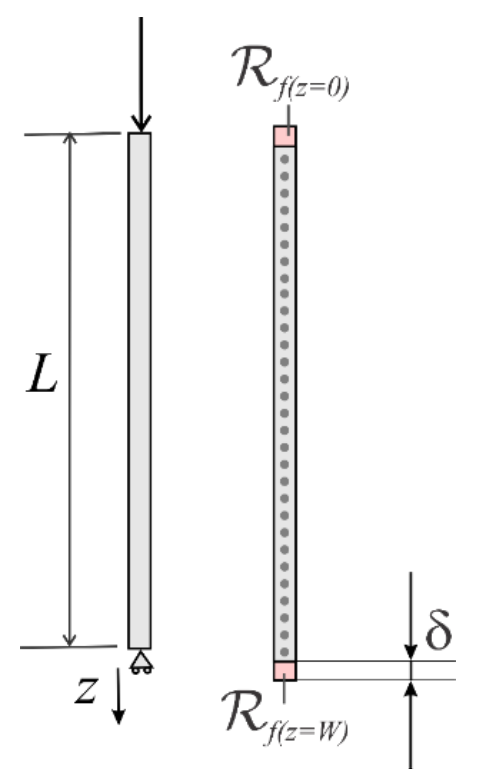

Fig. 2. Geometry and discretization of the one-dimensional consolidation problem.

Boundary conditions are imposed as

$$
\begin{aligned}
& \sigma_{z z}(z=0, t)=-P_{o} \\
& u_{z}(z=L, t)=0 .
\end{aligned}
$$

Also, the fluid drainage is only allowed at the loaded end $(z=0)$. Thus, the boundary conditions on the pressure field are imposed as

$$
P(z=0, t)=0
$$

and

$$
\frac{\partial P}{\partial z}(z=L, t)=0
$$

The initial conditions for the pore water pressure and the instantaneous displacement caused by the applied pressure $P_{o}$ are specified as 


$$
\begin{aligned}
& P(z, t=0)=v P_{o} \\
& u_{z}(z, t=0)=a_{i} P_{o}(h-z)
\end{aligned}
$$

where

$$
v=\frac{a-a_{i}}{\alpha a}
$$

in which

$$
a_{i}=\frac{a}{1+\alpha^{2} a Q}
$$

with $a^{-1}=1.0 \times 10^{8} \mathrm{~N} / \mathrm{m}^{2}$ (inverse final compressibility), and the Biot parameter, $\alpha=0.5$.

During the analysis, these boundary conditons are imposed at material points inside a fictitious region, $\mathcal{R}_{f}$.

The PD model is constructed by considering 1000 material points, and the time step size is chosen as $\Delta t=1 \times 10^{-6} \mathrm{~s}$.

The analytical solution to this problem derived by Wang (2000) is in the form

$$
P(z, t)=\frac{4 v P_{o}}{\pi} \sum_{m=0}^{N}\left\{\frac{1}{2 m+1} \exp \left(-\left(\frac{(2 m+1) \pi}{2 L}\right)^{2} c t\right) \times \sin \left(\frac{(2 m+1) \pi z}{2 L}\right)\right\}
$$

and 


$$
\begin{aligned}
u_{z}(z, t)= & c_{m} v P_{o}\left\{(L-z)-\frac{8 L}{\pi^{2}} \sum_{m=0}^{N}\left\{\frac{1}{(2 m+1)^{2}}\right\}\right\} \\
& \times \exp \left(-\left(\frac{(2 m+1) \pi}{2 L}\right)^{2} c t\right) \times \cos \left(\frac{(2 m+1) \pi z}{2 L}\right)+u_{z}(z, 0)
\end{aligned}
$$

where

$$
\frac{1}{c}=\frac{a \alpha^{2}}{\kappa}+\frac{1}{Q \kappa} \text { and } c_{m} v=a-a_{i}
$$

As shown in Figs. 3 and 4, there is a good agreement between peridynamic and analytical results. As a result of the applied external loading $P_{o}$ and corresponding water drainage, the pore pressure decreases and the deformation of soil increases as time progresses. As the water is squeezed out and the size of the soil column decreases, the soil consolidates.

Moreover, an error analysis is also performed. L2 error norms for both displacement and pore pressure are obtained as shown in Figs. 5a,b. L2 error norms for both displacement and pore pressure decrease as the number of materials points increases, in other words, as the distance between material points decreases.

\subsection{Two-dimensional consolidation}

This case concerns the extension of the one-dimensional consolidation problem to two dimensions. The length and width of the region (Fig. 6) are specified as $L=15 \mathrm{~m}$ and $W=1.5 \mathrm{~m}$, respectively. Loading and drainage conditions are the same as those of the preceding problem. Also, no flow is allowed from the lower and lateral surfaces. In addition, the lower surface is constrained in the vertical direction while the lateral surfaces are constrained in the horizontal direction. 


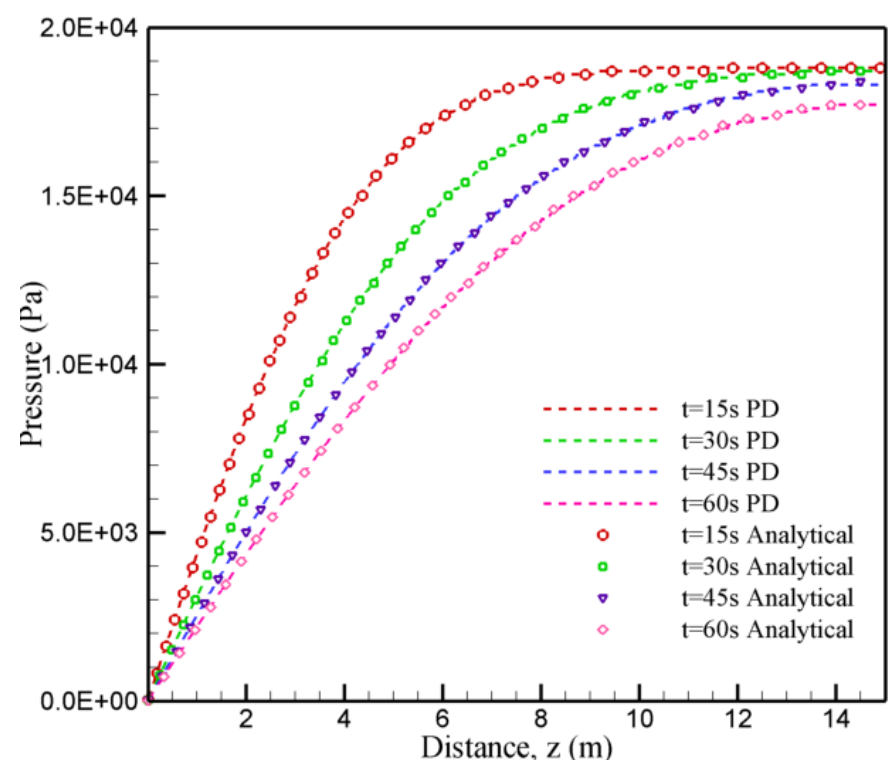

Fig. 3. Comparison of analytical and PD pore pressure solutions along the region.

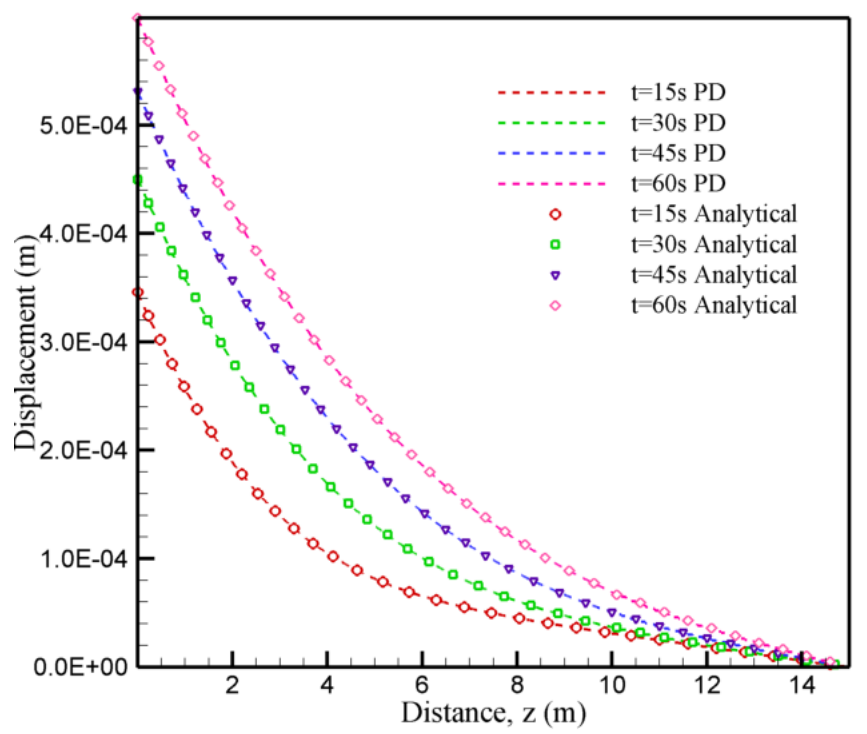

Fig. 4. Comparison of analytical and PD vertical displacements along the region. 


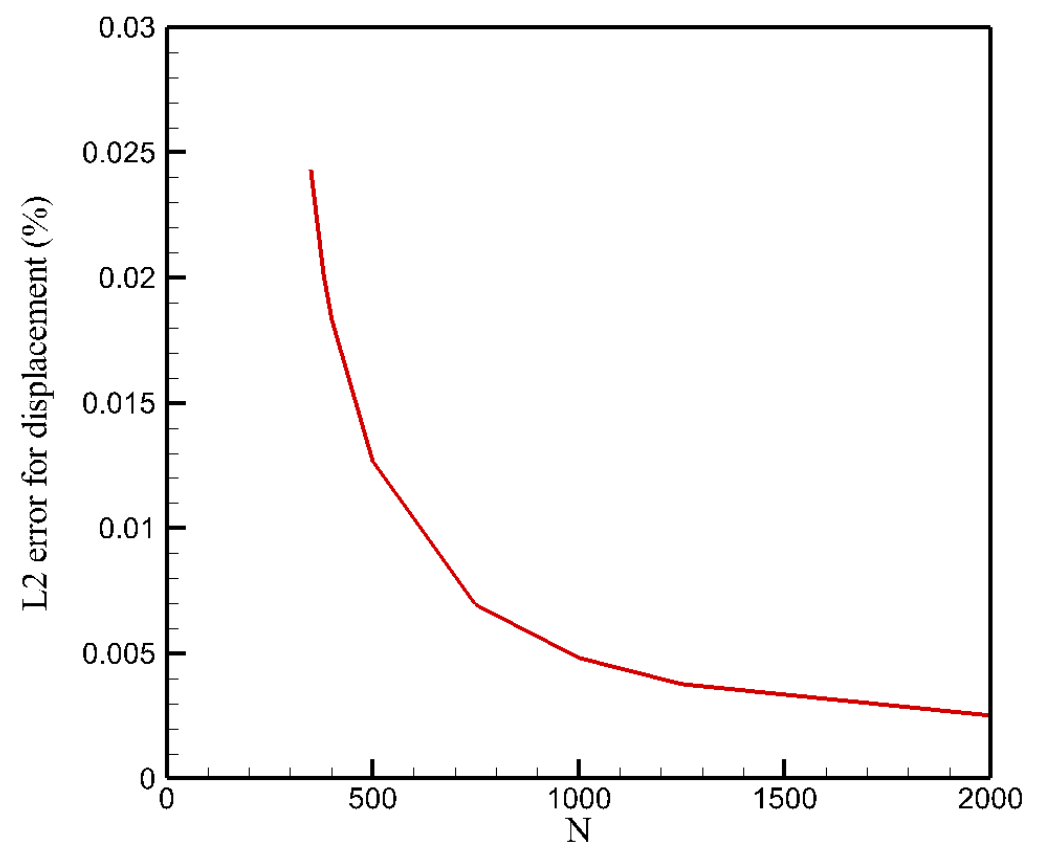

(a)

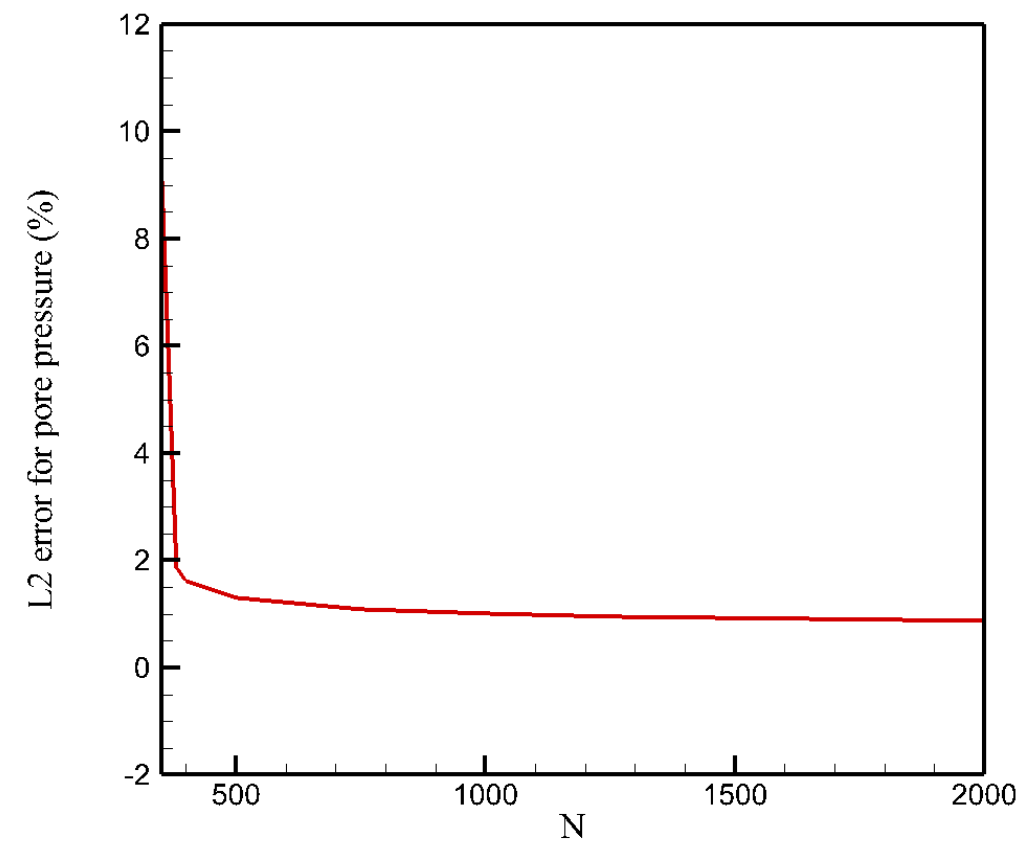

(b)

Fig. 5. L2 error norm variation as the number of material points, $N$, increases (a) displacement, (b) pore pressure [\%]. 


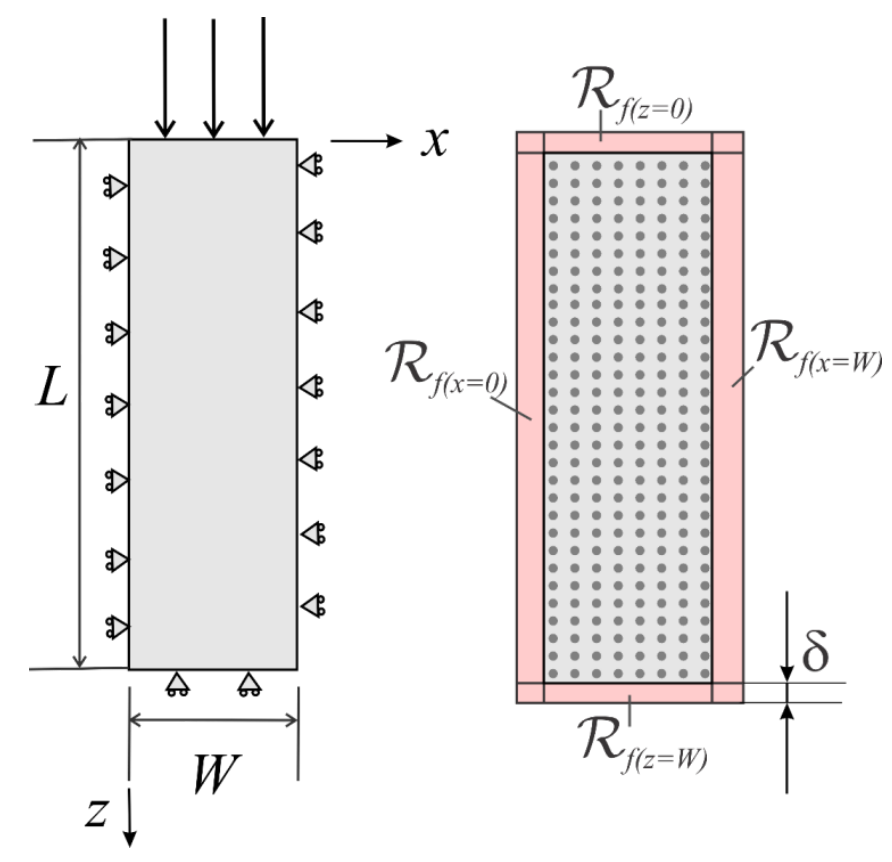

Fig. 6. Geometry and discretization of the two-dimensional consolidation problem.

Additional boundary conditions arising from two-dimensional consideration are

$$
\begin{aligned}
& u_{x}(x=0, z, t)=0 \\
& u_{x}(x=W, z, t)=0 \\
& \frac{\partial P}{\partial x}(x=0, z, t)=0 \\
& \frac{\partial P}{\partial x}(x=W, z, t)=0 .
\end{aligned}
$$

The PD model is constructed by considering $200 \times 20$ material points in the $z$ - and $x$ - directions, respectively. The time step size is chosen as $\Delta t=1 \times 10^{-5} \mathrm{~s}$.

The PD predictions are verified against FEA results generated by using ANSYS. The vertical displacements and pore pressure distributions predicted at $t=0.2 \mathrm{~s}, t=1.0 \mathrm{~s}$, and $t=2.0 \mathrm{~s}$ are shown in Figs. 7 and 8, respectively. The comparison of the PD predictions 
with ANSYS indicate good agrement. The variation of the vertical displacement and the pore pressure in the form of contours are presented in Fig. 9.

Similar to the results obtained for the 1-D solution, due to the applied external loading, the water pressure dissipates, as shown in Fig. 7. As the water drains, the soil deforms even more due the applied external loading condition. The deformation increases as the time progresses as shown in Fig. 8.

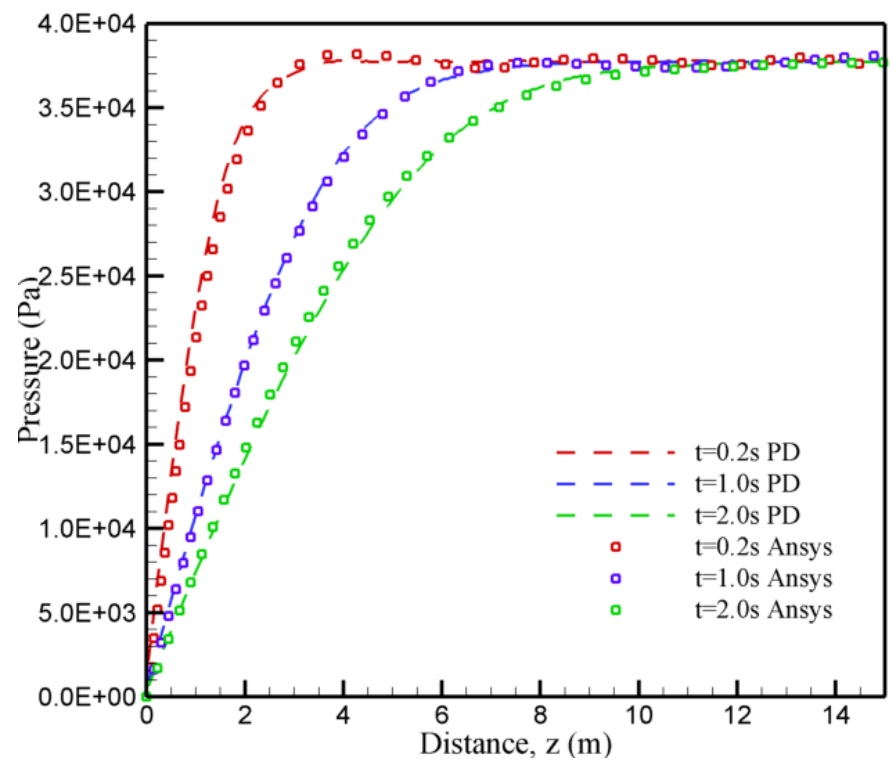

Fig. 7. Comparison of pore pressure between PD and ANSYS solutions.

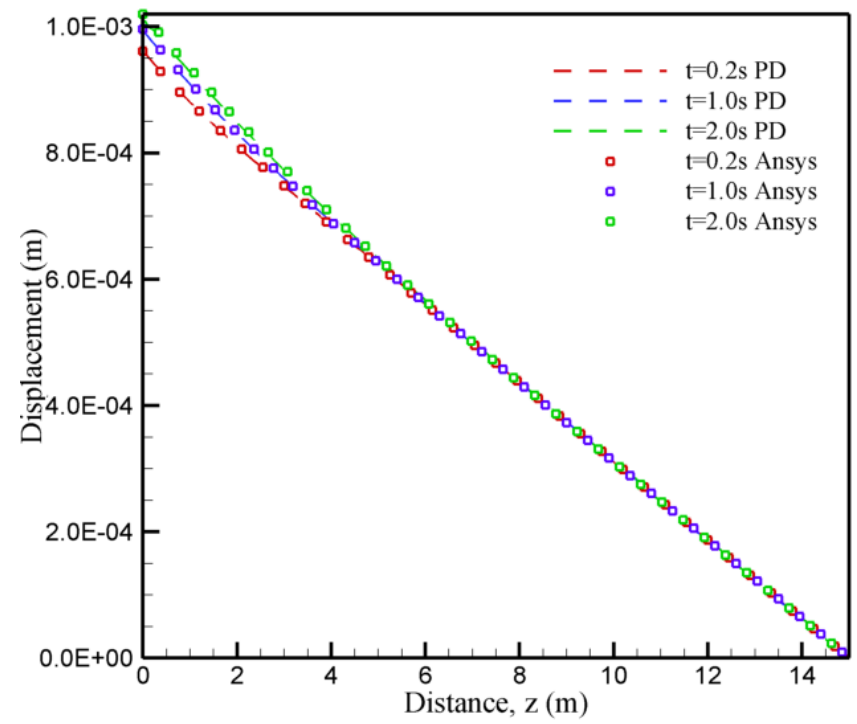

Fig. 8. Comparison of vertical displacement between PD and ANSYS solutions. 


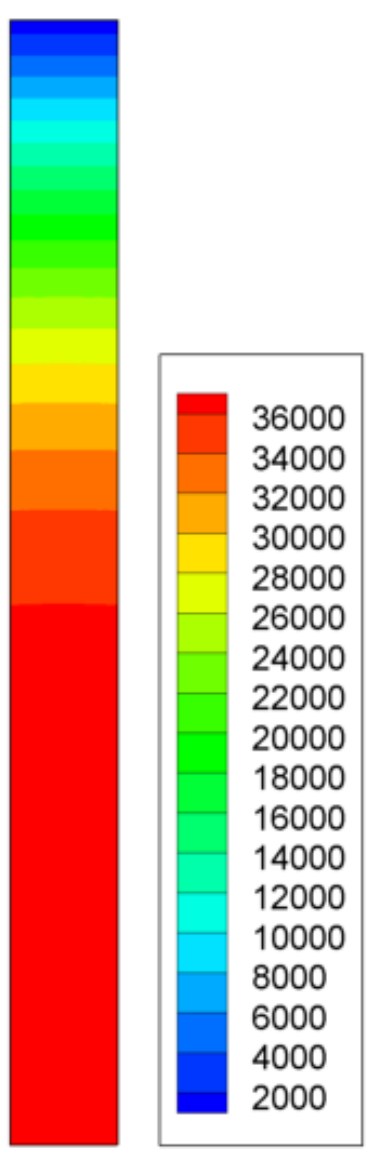

(a)

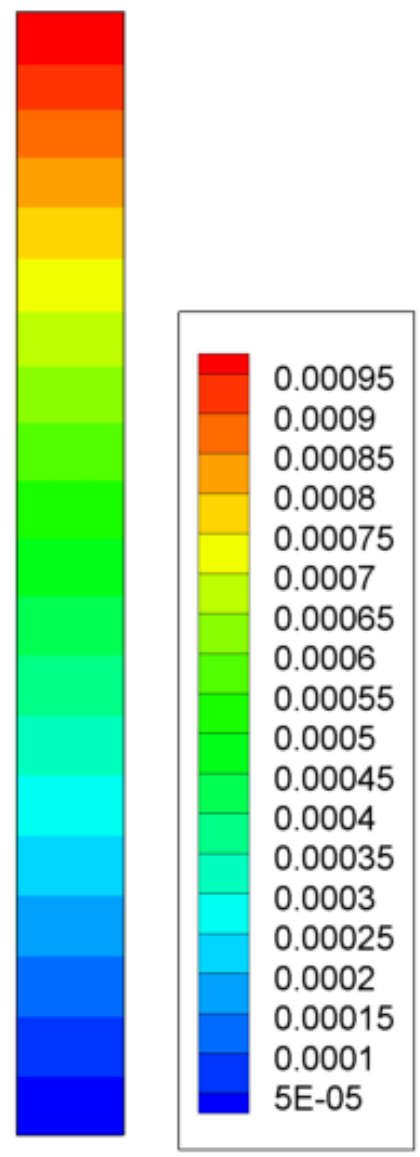

(b)

Fig. 9. PD solutions at $t=2.0 \mathrm{~s}$ : (a) fluid pressure (Pa); (b) vertical displacement (m).

\subsection{A hydraulically pressurized crack}

Finally, a hydraulically pressurized crack case is considered. The geometry of the region with a pre-existing crack is described in Fig. 10. The extent of the region is specified by $L=W=6 \mathrm{~m}$, and the crack length is $2 a=0.3 \mathrm{~m}$. The crack is exposed to a hydraulic pressure of $P_{o}$ on its surfaces. In addition, all surfaces of this region are constrained and no flow is allowed. Therefore, the boundary conditions on the lateral surfaces are specified as

$$
u_{x}(x=0, z, t)=0
$$




$$
\begin{aligned}
& u_{x}(x=W, z, t)=0 \\
& u_{z}(x, z=0, t)=0 \\
& u_{z}(x, z=L, t)=0 \\
& \frac{\partial P}{\partial x}(x=0, z, t)=0 \\
& \frac{\partial P}{\partial x}(x=W, z, t)=0 \\
& \frac{\partial P}{\partial z}(x, z=0, t)=0 \\
& \frac{\partial P}{\partial z}(x, z=L, t)=0 .
\end{aligned}
$$

The boundary conditions on the crack surface are imposed as

$$
\begin{aligned}
& \sigma_{z z}(z=L / 2, x)=-P_{o} \frac{t}{t_{o}} \quad L / 2-a<x<L / 2+a \\
& P(z=L / 2, x)=P_{o} \frac{t}{t_{o}} \quad L / 2-a<x<L / 2+a
\end{aligned}
$$

where $P_{o}=2000 \mathrm{~Pa}$ and $t_{o}=0.01 \mathrm{~s}$. The initial conditions are specified as

$u_{x}(x, z, t=0)=0$

$u_{z}(x, z, t=0)=0$

$u_{x}(x, z, t=0)=0$

$u_{z}(x, z, t=0)=0$

$P(x, z, t=0)=0$

Unlike the previous problems, the Biot parameter is assigned a value of $\alpha=0.1$. Grid spacing of $\Delta=0.015 \mathrm{~m}$ leads to a grid size of $400 \times 400$ in the peridynamic model, and a time step size of $\Delta t=1 \times 10^{-6} s$ is employed in the analysis. Initially, the crack is not allowed to propagate and the displacement and pore pressure fields are predicted. The 
corresponding displacement components are shown in Figs. 11 and 12. Negative dilatation develops near the upper and lower crack surfaces because of the applied pressure. However, dilatation is positive in the regions ahead of the crack tips arising from the opening mode of deformation. Therefore, there exists pressure rise in the regions of negative dilatation, and pressure drop in the regions of positive dilatation, as shown in Fig. 13. The verification of the PD predictions is established by comparing with ANSYS solutions at $t=0.01 \mathrm{~s}$, as shown in Figs. 11-13.

Next, the crack is allowed to propagate and the applied pressure is extended to the newly created fracture surfaces. The critical stretch value of the material is specified as $s_{c r}=3 \times 10^{-6}$. As the crack grows, the regions of negative and positive dilatation grow. Thus, the pore pressure increases further near the upper and lower crack surface and decreases near the crack tips, forming two sinks, as shown in Fig. 14. As expected, the crack propagates in a self-similar manner.

\section{Conclusions}

This study demonstrates a new fully coupled poroelastic peridynamic formulation. Peridynamics does not suffer from several issues that classical continuum mechanics based approaches confronted including remeshing and mesh dependency. The formulation is validated by considering one- and two-dimensional consolidation problems. Peridynamic results agree well with analytical and numerical solutions. Moreover, peridynamic analysis of a hydraulically pressurized crack is demonstrated. Peridynamic results are compared against finite element analysis results by considering a stationary crack and a good agreement is observed between the two solutions. Then, the crack is allowed to propagate and fluid pore pressure distributions are obtained as the crack propagates. Based on the evaluated results, it can be concluded that the current peridynamic formulation has a potential to be used for the analysis of more sophisticated poroelastic problems and can be extended to be applicable for more complex material behavior. 


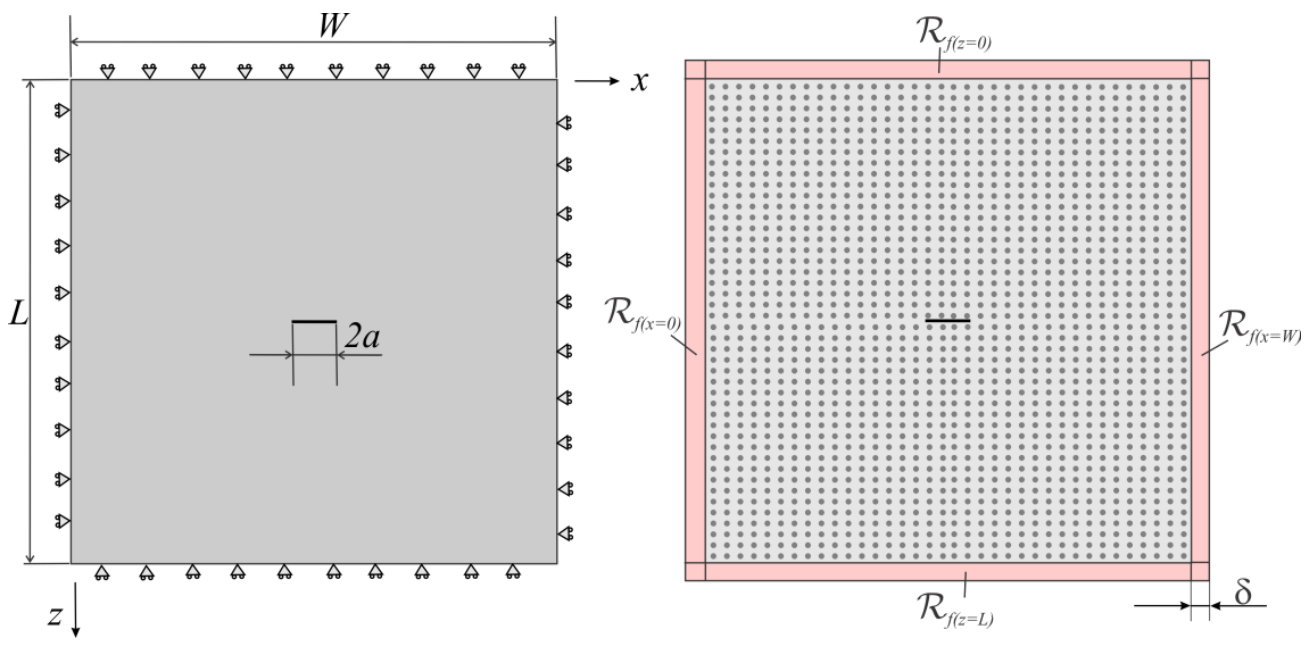

Fig. 10. Description of domain geometry (left) and discretization (right) for a hydraulically pressurized crack problem.

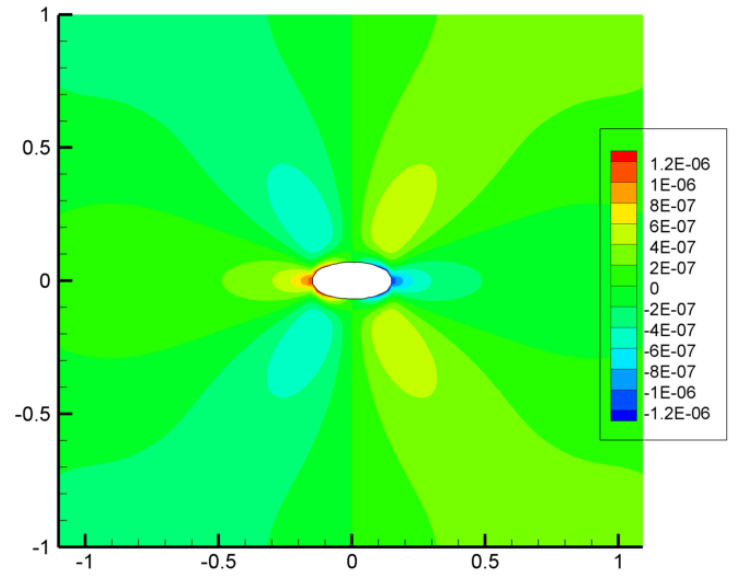

(a)

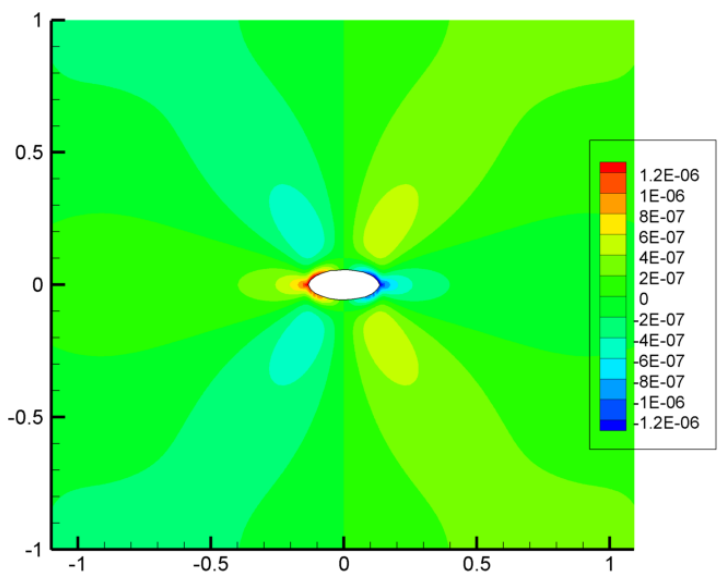

(b)

Fig. 11. Horizontal displacement (m) PD solution at $t=0.01 s$ : (a) PD; (b) ANSYS solution (deformed configuration with a magnification of $10^{4}$ ). 


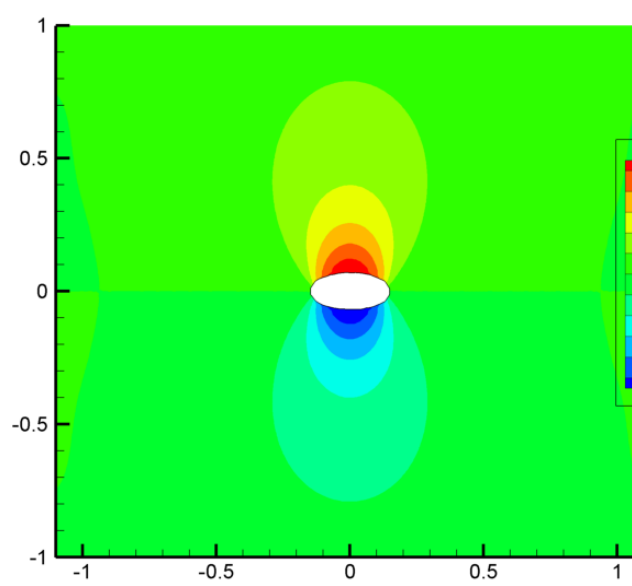

(a)

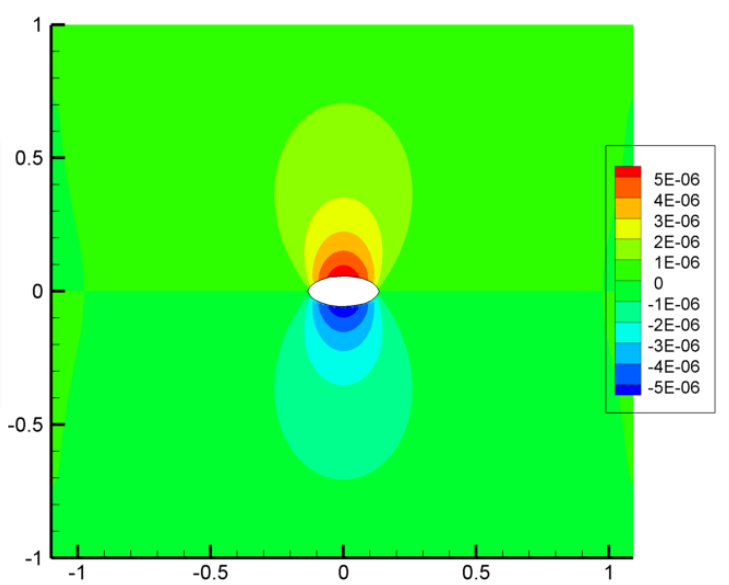

(b)

Fig. 12. Vertical displacement (m) PD solution at at $t=0.01 s$ : (a) PD; (b) ANSYS solution (deformed configuration with a magnification of $10^{4}$ ).

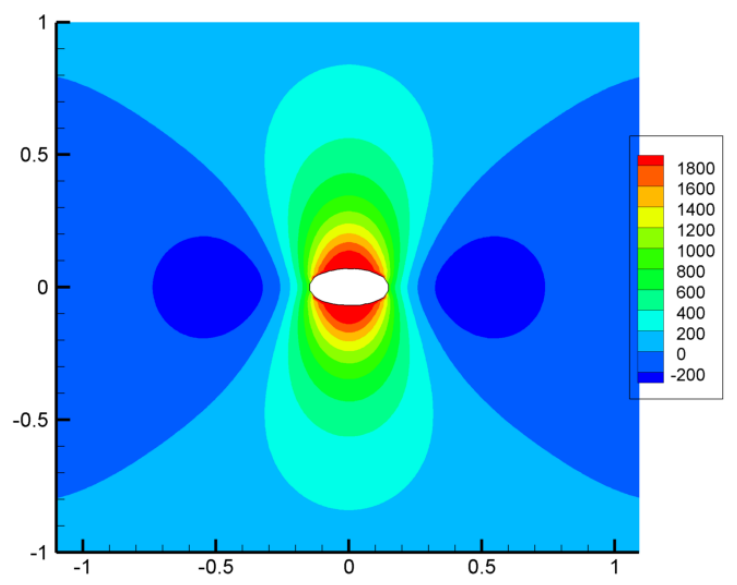

(a)

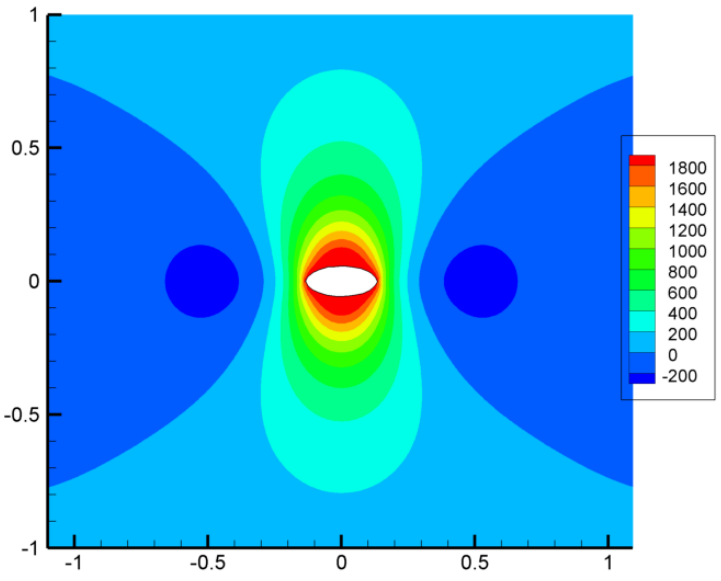

(b)

Fig. 13. PD fluid pore pressure predictions (Pa) at $t=0.01 s$ : (a) PD; (b) ANSYS solution (deformed configuration with a magnification of $10^{4}$ ). 


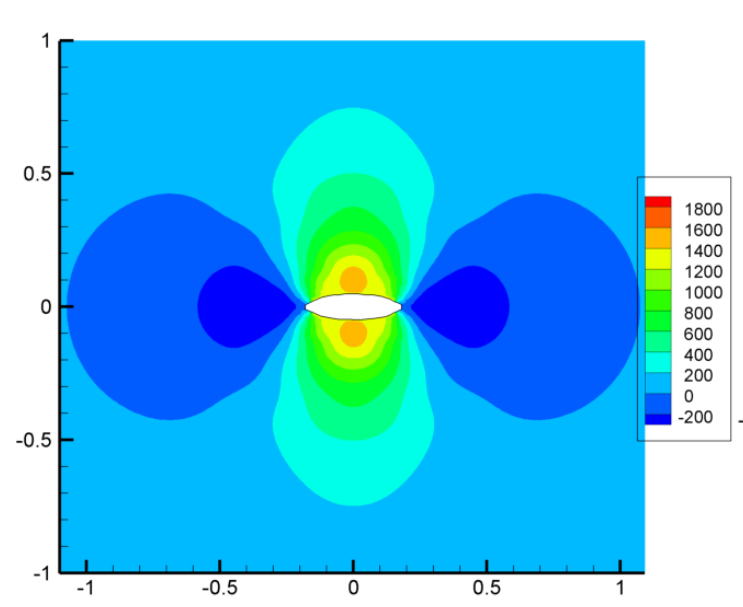

(a)

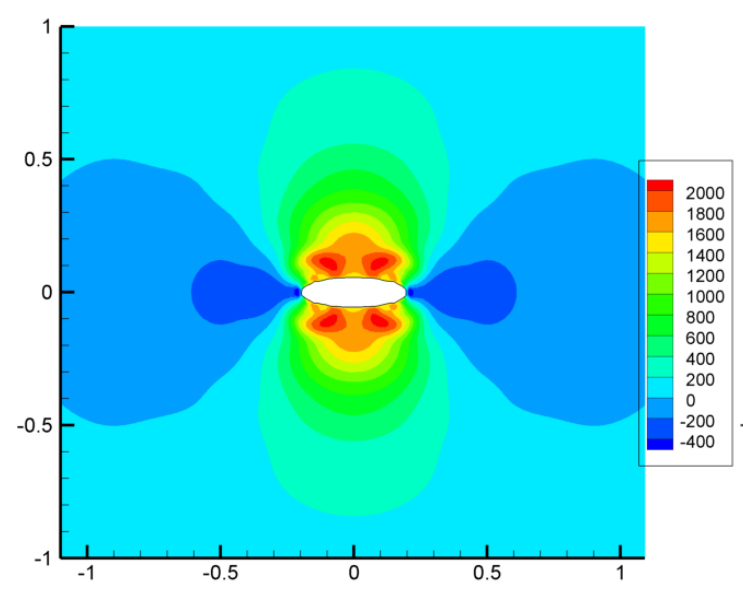

(c)

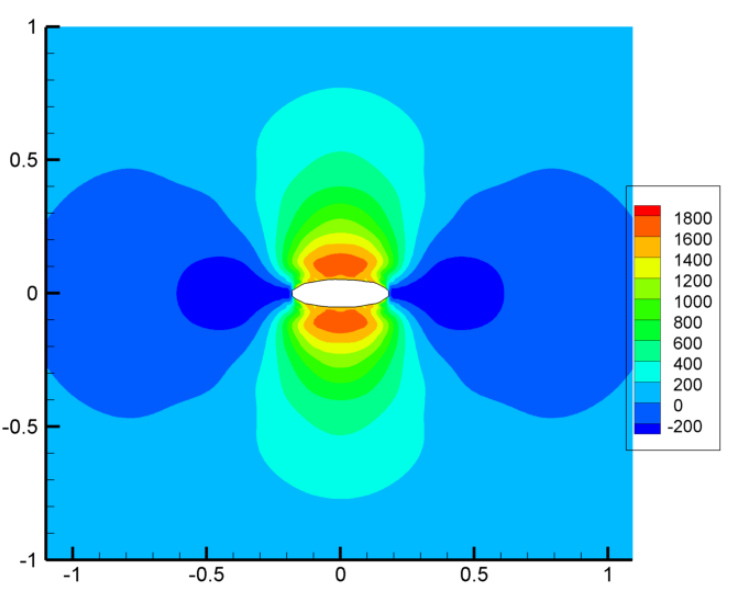

(b)

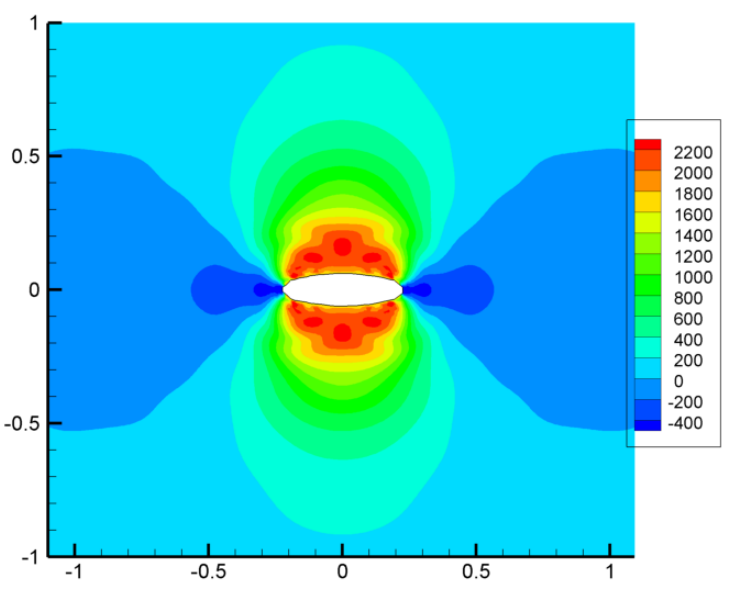

(d)

Fig. 14. PD solution of fluid pore pressures with crack propagation ( $\mathrm{Pa}$ ) around the crack:

(a) $t=0.0065 \mathrm{~s}, 2 a=0.375$; (b) $t=0.007 \mathrm{~s}, 2 a=0.375$; (c ) $t=0.0075 \mathrm{~s}, 2 a=0.405$; (d) $t=0.008 s, 2 a=0.465$ (deformed configuration with a magnification of $10^{4}$ ).

\section{References}

Armero, F., Simo, J.C., 1992. A new unconditionally stable fractional step method for nonlinear coupled thermomechanical problems. Int. J. Numer. Meth. Eng. 35, 737766.

Biot, M.A., 1941. General theory of three-dimensional consolidation. J. Appl. Phys. 12, $155-164$.

Bobaru, F., Duangpanya, M., 2010. The peridynamic formulation for transient heat conduction. Int. J. Heat Mass Tran. 53, 4047-4059.

Bobaru, F., Duangpanya, M. A peridynamic formulation for transient heat conduction in bodies with evolving discontinuities. J. Comput. Phys. 231, 2764-2785.

Carcione, J. M., Morency, C., Santos, J. E., 2010. Computational poroelasticity - A review. Geophysics 75 (5), 75A229-75A243. 
Chen, Z., 2012. Finite element modelling of viscosity-dominated hydraulic fractures. J. Pet. Sci. Eng. 88-89, 136-144.

Chen, Z., Bobaru, F., 2015. Peridynamic modeling of pitting corrosion damage. J. Mech. Phys. Solids 78, 352-381.

De Meo, D., Diyaroglu, C., Zhu, N., Oterkus, E., Siddiq, M.A., 2016. Modelling of stress-corrosion cracking by using peridynamics. Int. J. Hydrogen Energ. 41, 65936609.

Farhat, C., Park, K.C., Duboispelerin, Y., 1991. An unconditionally stable staggered algorithm for transient finite-element analysis of coupled thermoelastic problems. Comput. Methods Appl. Mech. Eng. 85, 349-365.

Gerstle, W., Silling, S., Read, D., Tewary, V., Lehoucq, R., 2008. Peridynamic simulation of electromigration, Comp. Mater. Contin. 8, 75-92.

Han, S. W., Diyaroglu, C., Oterkus, S., Madenci, E., Oterkus, E., Hwang, Y., Seol, H., 2016. Peridynamic Direct Concentration Approach by Using ANSYS. IEEE Electronic Components and Technology Conference (ECTC), 544-549.

Han, S., Lim, S., Bae, J., Hwang, Y., Lee, S., Oterkus, S., Madenci, E., Diyaroglu, C. and Oterkus, E., 2015. Equivalent acceleration assessment of JEDEC moisture sensitivity levels using peridynamics. IEEE 65th Electronic Components and Technology Conference (ECTC), 1518-1523.

Helmons, R. L. J., Miedema, S. A., Alvarez Grima, M. and van Rhee, C., 2016. Modeling fluid pressure effects when cutting saturated rock. Eng. Geol. 211, 50-60.

Hunsweck, M.J., Shen, Y., Lew, A.J., 2013. A finite element approach to the simulation of hydraulic fractures with lag. Int. J. Numer. Anal. Methods Geomech. 37, 9931015.

Kilic, B., Madenci, E., 2010. Peridynamic theory for thermomechanical analysis. IEEE Trans. Adv. Packag. 33, 97-105.

Lecampion, B., 2009. An extended finite element method for hydraulic fracture problems. Commun. Numer. Methods in Eng. 25, 121-133.

Li, Y., Chen, Y.-F. and Zhou, C.-B., 2014. Hydraulic properties of partially saturated rock fractures subjected to mechanical loading. Eng. Geol. 179, 24-31.

Liu, W.K., Chang, H.G., 1985. A note on numerical-analysis of dynamic coupled thermoelasticity. ASME J. Appl. Mech. 52, 483-485.

Madenci, E., Oterkus, E., 2014. Peridynamic Theory and Its Applications. Springer, Boston, MA.

Madenci, E., Oterkus, S., 2016. Ordinary state-based peridynamics for plastic deformation according to von Mises yield criteria with isotropic hardening. J. Mech. Phys. Solids 86, 192-219.

Oterkus, S., 2015. Peridynamics for the solution of multiphysics problems. PhD Thesis, University of Arizona.

Oterkus, E., Madenci, E., 2011. Damage prediction in graphene thermoplastics for potential electronic packaging applications. IEEE 61st Electronic Components and Technology Conference (ECTC), 2134-2140.

Oterkus, S., Madenci, E., 2013. Crack growth prediction in fully-coupled thermal and deformation fields using peridynamic theory. 54th AIAA/ASME/ASCE/AHS/ASC Structures, Structural Dynamics and Materials Conference Boston, MA. 
Oterkus, S., Madenci, E., 2014. Peridynamics for fully coupled thermomechanical analysis of fiber reinforced laminates. 55th AIAA/ASME/ASCE/AHS/ASC Structures, Structural Dynamics and Materials Conference, National Harbor, MD.

Oterkus, S., Madenci, E., 2015. Peridynamics for antiplane shear and torsional deformations. J. Mech. Phys. Solids 10, 167-193.

Oterkus, S., Fox, J., Madenci, E., 2013. Simulation of electromigration through peridynamics. IEEE 63rd Electronic Components and Technology Conference (ECTC), Las Vegas, NV.

Oterkus, S., Madenci, E., Agwai, A., 2014a. Fully coupled peridynamic thermomechanics, J. Mech. Phys. Solids 64, 1-23.

Oterkus, S., Madenci, E., Agwai, A., 2014b. Peridynamic thermal diffusion. J. Comput. Phys. 265, 71-96.

Oterkus, S., Madenci, E., Oterkus, E., Hwang, Y., Bae, J., Han, S., 2014c. Hygro-thermomechanical analysis and failure prediction in electronic packages by using peridynamics, IEEE 64th Electronic Components and Technology Conference, Lake Buena Vista, FL.

Rabczuk, T., Belytschko, T., 2004. Cracking particles: a simplified meshfree method for arbitrary evolving cracks. Int. J. Numer. Meth. Eng. 61, 2316-2343.

Rabczuk, T., Belytschko, T., 2007. A three-dimensional large deformation meshfree method for arbitrary evolving cracks. Comput. Methods Appl. Mech. Eng. 196, 2777-2799.

Rabczuk, T., Gracie, R., Song, J-H, Belytschko, T., 2010a. Immersed particle method for fluid-structure interaction. Int. J. Numer. Meth. Eng. 81, 48-71.

Rabczuk, T., Zi, G., Bordas, S., Nguyen-Xuan, H., 2010b. A simple and robust threedimensional cracking-particle method. Comput. Methods Appl. Mech. Eng. 199, 2437-2455.

Ren, H., Zhuang, X., Cai, Y., Rabczuk, T., 2016. Dual-horizon peridynamics. Int. J. Numer. Meth. Eng. 108, 1451-1476.

Silling, S.A., 2000. Reformulation of elasticity theory for discontinuities and long-range forces. J. Mech. Phys. Solids 48, 175-209.

Silling, S.A., Askari, E., 2005. A meshfree method based on the peridynamic model of solid mechanics. Comput. Struct. 83, 1526-1535.

Terzaghi, K., 1925. Principle of soil mechanics. Erdbaumechanik auf Bodenphysikalischer Grundlage.

Turner, D.Z., 2013. A non-local model for fluid-structure interaction with applications in hydraulic fracturing. Int. J. Comput. Methods Eng. Sci. Mech. 14, 391-400.

Wang, H.F., 2000. Theory of Linear Poroelasticity with Applications to Geomechanics and Hydrogeology. Princeton University Press, Princeton, NJ.

Zhou, X. and Burbey, T. J., 2014. Deformation characteristics of a clayey interbed during fluid injection. Eng. Geol. 183, 185-192. 